\title{
A rich vehicle routing problem dealing with perishable food: a case study
}

\author{
Pedro Amorim ${ }^{\S}$ Sophie N. Parragh ${ }^{\star}$ \\ Fabrício Sperandio ${ }^{\S}$ Bernardo Almada-Lobo ${ }^{\S}$ \\ ${ }^{\S}$ INESC TEC, Faculdade de Engenharia, Universidade do Porto, \\ Rua Dr. Roberto Frias, s/n, 4600-001 Porto, Portugal. \\ \{amorim.pedro, almada.lobo\}@fe.up.pt \\ ${ }^{\star}$ Department of Business Administration, University of Vienna \\ Bruenner Strasse 72, 1210 Vienna, Austria \\ sophie.parragh@univie.ac.at
}

\begin{abstract}
This paper presents a successful application of operations research techniques in guiding the decision making process to achieve a superior operational efficiency in core activities. We focus on a rich vehicle routing problem faced by a Portuguese food distribution company on a daily basis. This problem can be described as a heterogeneous fleet site dependent vehicle routing problem with multiple time windows. We use the adaptative large neighbourhood search framework, which has proven to be effective to solve a variety of different vehicle routing problems. Our plans are compared against those of the company and the impact that the proposed decision support tool may have in terms of cost savings is shown. The algorithm converges quickly giving the planner considerably more time to focus on valueadded tasks, rather than manually correct the routing schedule. Moreover, contrarily to the necessary adaptation time of the planner, the tool is quite flexible in following market changes, such as the introduction of new customers or new products.
\end{abstract}

Keywords Vehicle Routing Problem, Adaptative Large Neighbourhood Search, OR in Industry, Decision Support Systems

\section{Introduction}

In Portugal, the farm-to-fork associated food industries generate a total sales value of over $€ 10.6$ billion and a total services value of $€ 150$ million, corresponding to 7.2 percent of the national gross domestic product (Instituto Nacional de Estatítica, 2011). Within this value chain, half of the amount comes from the production and the other half from the distribution activities. Distribution companies face several typical problems, at different hierarchical decision levels. For example, on a tactical level, the set of logistics providers to select and with which kind of contract, or on which days the clients should be visited, which is stipulated upon a distribution calendar contract; on a more operational level, there is the daily problem of designing the routes to serve customers previously assigned to that day based on their demand orders. This last problem is the focus of this case-study.

This work studies a real-life problem faced by a Portuguese food distribution company that supplies a wide range of food solutions for a diverse range of clients. A food solution is defined

This is the peer reviewed version of the following article:

Amorim, P., Parragh, S. N., Sperandio, F., Almada-Lobo, B. (2014). A rich vehicle routing problem dealing with perishable food: a case study. Top, 22(2), 489-508.

The final publication is available at Springer via http://dx.doi.org/10.1007/s11750-012-0266-4 


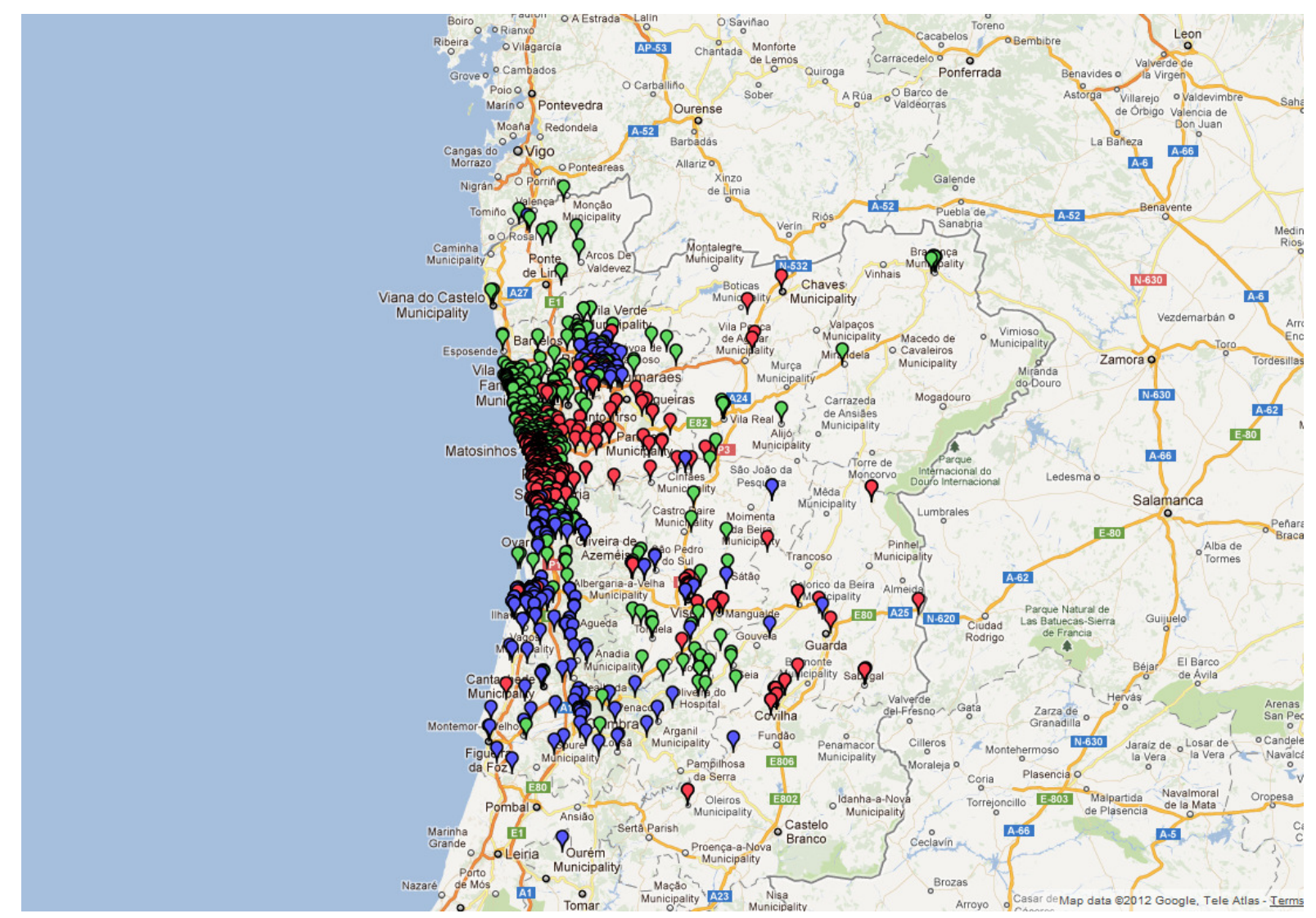

Figure 1: Geographical location of customers in the north filial

as a service that provides a quick response to customer orders for a set of food products. The vision of the company is to be the best operating in the business in terms of quality and it wants to achieve high operational efficiency. The company has two distribution platforms with decentralized operations management and more than 50 multi-temperature vehicles that travel more than 3 million kilometres per year. With around 15 thousand references of products, it moves more than 45 thousand tons per year. These food products may be of various types and in many different quantities. They vary from pallets of beer to small baskets with rice, milk and potatoes. Despite having more than 200 people working for them and the fact that the distribution is their core activity, only four people are responsible for the routings as they heavily rely on the scheduling software. The north filial (the one we are working with) has in their portfolio around 1570 active clients spread in the north of Portugal (Figure 1). Their market ranges from primary schools, to prisons, banks and wholesalers.

The distribution business of this company is affected by high seasonality throughout the year. This situation could demand significant fixed and maintenance costs related to the fleet management. These costs would be hardly diluted in the operational efficiency, since the company would need to have a fleet dimensioned for the peak season that would result in a low return on assets. To overcome such scenario and increase the operational flexibility, the company decided to contract with a pool of third party logistics providers upper and lower levels on the number of vehicles of various kinds (in size and compartments) that have to be available to the company within a twelve hour window. In practice, this means that having customer orders fixed the day before, the company is able to create the routing plan and know precisely how many and which type of vehicles are needed from the providers.

By looking at the descriptions of the company and of the main problem under consideration, it is clear that an optimized daily routing yields a considerable impact in a company that has as core activity the distribution of food. In fact, a reduction of only $5 \%$ in the total distance 
travelled corresponds to 150 thousand fewer kilometers travelled each year. With ever rising petrol prices the decision made for such an operational tool has certainly strategic impacts. Through a set of meetings with the company's employees and executives, five main practical motivations to carry out this work are found. First, planned routes are almost fixed from day to day and only small adjustments are performed as the planner sees post-processing opportunities, resulting in sub-optimal solutions. Second, whenever the senior planner is not at work, the company plans suffer a considerable quality decrease. Third, this planner has other functions in the company that are seriously jeopardized by the tremendous amount of time (4 hours per day on average) that he spends improving the generated plans. Fourth, whenever there is a disruptive happening in the amount of clients to be dealt with, the planner needs some weeks to adapt to the new situation and, meanwhile, the plans are not of the same quality. Fifth, there is a willing to cut distribution costs.

The operational routing problem, which the planner has to deal with, can be seen as an extension of the vehicle routing problem (VRP). The VRP is a problem in which a set of vehicles with the same capacity (homogeneous fleet) is initially located at a depot. These vehicles shall visit a set of customers exactly once and both their locations and demands are known. The objective is to minimize the total travel costs and/or vehicles used to visit all customers. The demand fulfilled in each route can not exceed the capacity of the vehicle and all routes have to end at the depot. The VRP is a NP-hard problem (Savelsbergh, 1985) and to solve real-world instances approximate solution methods, such as metaheuristics have to be used. The routing problem faced by the company relaxes several of the assumptions imposed by the traditional VRP and includes some specific characteristics, making the overall problem more demanding.

The company needs to fulfil customers demand on a daily basis for an assortment of products of different temperature requirements, categories and quantities. In perishable food distribution (Amorim et al, 2011), products have different temperature requirements to avoid spoilage during routing and to conserve the organoleptic proprieties of the products. Basically, the products can be split into three categories: dry, cold (fresh) and frozen. Therefore, vehicles equipped with compartments that can be set to different temperatures are employed. Consequently, the first generalization of the traditional VRP concerns the homogeneity of the fleet. In our problem, trucks can be divided according to their different temperature compartments in dry and refrigerated. On one hand, refrigerated trucks are able to carry dry, fresh and frozen foods (as no problem arises from transporting dry products at lower temperatures) and they are more expensive to operate. On the other hand, dry trucks can only transport food that has a stable behaviour at an ambient temperature. For the sake of competitiveness, food distribution companies, such as the one under study are able to choose upon different types of vehicles regarding their temperature capabilities. However, this is not the only factor inducing a heterogeneous fleet. Since customers are not only very heterogeneous in terms of business activities but also in terms of their accessibility conditions, there is a need for vehicles with different sizes. There are customers located on the side of the motorway, but others are in the very inner city center or in rural areas difficult to access. This means that large trucks may be forbidden to service a certain customer due to the impossibility of accessing the delivery site. Therefore, the company's fleet may be catalogued according to the respective temperature compartments and to the size of the trucks.

The different product categories yielding different temperature requirements enforce an extra relaxation of the traditional VRP regarding the imposition that each customer may only be visited exactly once, by one truck (no split-deliveries). In our case study, if a customer demands both dry and fresh/frozen products, then he may be serviced either by a sole refrigerated truck carrying all products or by a combination of two trucks where one carries the fresh/frozen products and the other the dry demand.

There is one more characteristic worth of mention that distinguishes our case study problem from the traditional VRP. Customers serviced during the day have multiple hard time windows 
to be serviced. Most of the times either customers demand to be serviced early in the morning (for example, hospitals) or they demand not to be serviced at lunch time (hence, a time window in the morning and another in the afternoon). There are also customers that demand to be serviced only at night. In practice the routing for such customers may be done independently from the day customers, since for servicing the night customers the trucks need to go back to the warehouse.

According to the proper literature nomenclature, this problem corresponds to a heterogeneous fleet site dependent vehicle routing problem with multiple time windows (HF-SD-VRPMTW).

Our paper has a threefold contribution. First, a state-of-the-art metaheuristic (the adaptative large neighboorhod search) is adapted to solve a problem that, to the best of our knowledge, has never been solved in an integrated manner. Second, we show the impact of efficient operations research techniques in considerably lowering operational costs for business core activities. Third, we assess and understand the vehicle routing business practice of our case study and disclose other opportunities to integrate such operational research techniques in similar environments.

The remainder of our paper is as follows. In the next section the literature about related problems is reviewed. In Section 3, a formal mathematical description of the problem at hands is given and in Section 4 we describe the algorithm used. Thereafter, in Section 5 our results are compared with the ones obtained by the company's software. Finally, some conclusions are drawn in Section 6.

\section{Literature Review}

In this section we review the literature on food distribution and on VRPs that share common features with our HF-SD-VRP-MTW.

In Jansen et al (1998) the authors investigate the importance of multi-compartment distribution for catering companies through simulation. The profile of the customers demand is very close to our case with a split in dry, fresh and frozen products. However, routing is not part of their research. The authors conclude that multi-compartment distribution gets more economical as the number of customers serviced decrease. Mullaseril et al (1997) deal with the problem of distributing food in a cattle ranch. The problem is formulated with a set of split delivery capacitated rural postman problems with time windows since different feeds have to be distributed in different trucks because no compartments exist to separate them. Tarantilis and Kiranoudis (2001), concentrating on the distribution of fresh milk, formulate the problem as a heterogeneous fixed fleet VRP. In Tarantilis and Kiranoudis (2002) a real-world distribution problem of fresh meat is solved as a multi-depot VRP. Faulin (2003) implements a hybrid method that uses a combination of heuristics and exact algorithms to find a solution of a VRP with constraints enforcing narrow time windows and strict delivery quantities. According to the authors, these delivery scenarios are usually the case in the agribusiness industry. Osvald and Stirn (2008) extend a heuristic proposed in a previous work to solve the problem of distributing fresh vegetables in which perishability represents a critical factor. The problem was formulated as a vehicle routing problem with time windows (VRP-TW) and time-dependent travel times. The objective function minimizes the distance and time travelled, the delay costs for servicing late a customer and the costs related with perishability. Hsu et al (2007) consider the randomness of the perishable food delivery process and present a stochastic VRP-TW model that is further extended to consider time-dependent travel times. The problem is then solved by a heuristic procedure. Chen et al (2009) integrate production scheduling with the VRP-TW for perishable food products. The price paid by the retailer to the transporter varies as the product more or less spoils. Afterwards, the integrated model is solved with an iterative scheme in which the production part is solved using the Nelder-Mead method and the distribution part is solved 
by a constructive heuristic followed by an improvement one. Ambrosino and Sciomachen (2006) describe a case-study that is rather similar to ours. The company that the authors study is a food company that has to deliver perishable food through the national highway. Their fleet is homogeneous and able to carry dry, fresh and frozen products. They formulate the problem as an asymmetric capacitated vehicle routing problem with split deliveries and use a cluster first-route second heuristic to solve it.

Through the review on the food distribution literature it is noticeable that most of the features of our problem were tackled, although in a separated manner. However, the site dependent and the multiple time windows extensions are yet to be tackled.

Nag et al (1998) are the first to study the site dependent vehicle routing problem (SD-VRP). In this work several simple heuristics are developed. Chao et al (1999) continue the work on the SD-VRP by proposing a new heuristic that is both tested on previous and new instances. Cordeau and Laporte (2001) show that the SD-VRP can be converted into a periodic vehicle routing problem (P-VRP) and they provide results obtained using a tabu search heuristic for the P-VRP presented in a previous paper. The vehicle routing problem with multiple time windows (VRP-MTW) appears to be one of the VRP extensions with less work devoted to it. Doerner et al (2008) develop exact and approximate algorithms for the pickup of perishable goods (blood) motivated by a real case of the Austrian Red Cross. In this problem customers have multiple interdependent time windows. More recently, Bitao and Fei (2010) develop an ant colony algorithm coupled with local search to solve a problem with the same features.

Summarizing, although most of the current research on the VRP focuses on extensions to it, there is no single work portraying all together the characteristics of our problem.

\section{Problem Statement and Mathematical Formulations}

The notation and formulations used in this section are based on the VRP-TW formulation proposed by Cordeau and Laporte (2001).

In our company's problem, a set $K$ of different fixed capacity vehicles $k=1, \ldots, m$, initially located at a depot, are available to deliver perishable food goods to a set $N$ of customers $i, j=1, \ldots, n$ through a set of $\operatorname{arcs} A$. The problem is defined on a directed graph $G=(V, A)$, with $V=N \cup\{0, n+1\}$, where the depot is simultaneously represented by the two vertices 0 and $n+1$ and, therefore, $|V|=n+2$. Hence, we use the word vertices to refer to all nodes in the network and customers when we are excluding the vertices related with the depot. Each possible arc $(i, j)$ has an associated time and distance. Since we have as input real data for time and distance (see Section 5) based on the Portuguese road network (differentiating between highways and national roads and prioritizing time over distance when finding the path between two vertices), the triangular inequality does not hold in terms of distance, however, it holds in terms of time. Hence, although travelling from $i$ to $j$ is always faster, than passing by customer $c$ in between. It is not true that travelling from $i$ to $j$ is necessarily shorter, than passing intermediately through customer $c$. Each customer has a demand that needs to be satisfied for a certain number of products that may be dry, fresh or frozen. Moreover, customers want their requests available within hard time windows that can be more than one throughout the day and they need a certain time to be served that is dependent on the demand.

A feasible solution for this problem implies a collection of routes that correspond to paths starting at vertex 0 and ending at vertex $n+1$. These routes have to ensure that each customer is visited exactly once by one of the vehicles allowed for the service, satisfying simultaneously its demand and time windows. Furthermore, the cumulative demand of all customers that each vehicle serves can not exceed its capacity.

In order to have such a definition for a feasible solution and to decrease the complexity of the mathematical formulation, three preprocessing steps are performed. Firstly, service times are assumed to be function of the demand of each client. Hence, for each $100 \mathrm{~kg}$ that have to be 
delivered at a customer, 5 minutes are needed. As the demand is known before performing the routing, all service times can be calculated beforehand. Secondly, regarding the site dependency characteristic of the problem some adjustments may also be performed. Let $K t_{i}$ denote the set of vehicles that are able to serve $i$ regarding its demand temperature requirements and $K l_{i}$ denote the set of vehicles that are able to serve $i$ regarding its accessibility conditions. In the preprocessing it is possible then to find $K_{i}=K t_{i} \cap K l_{i}$ as the set of vehicles able to serve $i$. Thirdly, because vehicles can either transport only dry products or products of all temperature requirements, the company is able to gain some extra flexibility in the search space and, thus, a potential improvement in the objective function, by allowing a customer to be serviced by two vehicles if he requires dry and fresh and/or frozen products. This is a particular case of split delivery where one allows a client to be serviced by different trucks depending on the demanded temperature requirements. However, each delivery has to contain all the demand for a certain temperature requirement. Our procedure to tackle this situation in the preprocessing is as follows: if customer $i$ has demand for both dry and either fresh and/or frozen products, then this customer is split into $i^{\prime}$ and $i^{\prime \prime}$. These two new dummy customers have the same location as $i$. The demand of $i^{\prime}$ adds up for all dry products and the demand of $i^{\prime \prime}$ aggregates the demand for fresh and frozen products. Service times for these dummy customers are calculated based on the split demand. Customer $i^{\prime}$ has no special requirements regarding vehicle temperature compartments, i.e. $K_{i^{\prime}}=K l_{i}$. However, for $i^{\prime \prime}$ we have $K_{i^{\prime \prime}}=K t_{i^{\prime \prime}} \cap K l o c_{i}$. Thus, $i^{\prime}$ and $i^{\prime \prime}$ can be serviced at the same time by the same vehicle if it carries the dry products along with the fresh and frozen ones. Alternatively, it may be serviced by different trucks: one carrying the dry products and the other the remaining products.

The goal of the company is to minimize total costs. These costs correspond to variable travel costs, renting vehicle costs and driver costs. Regarding driver costs these are calculated based on the route duration. The logistic provider delivers each vehicle with a driver that can drive up to the regulated 8 hours. If the total time of a route surpasses such legal limit, a new driver has to accompany the main one yielding an extra cost.

We are now able to define the mathematical formulations for the HF-SD-VRP-MTW. We use the following indices, parameters, and decision variables.

\section{Indices}

$k \quad$ vehicles

$i, j \quad$ vertices after preprocessing

$v \quad$ time windows

\section{Sets and Parameters}

$K_{i} \quad$ set of vehicles able to serve vertex $i$

$T W_{i} \quad$ set of time-windows on vertex $i$

$C^{k} \quad$ Capacity of vehicle $k$

$s_{i} \quad$ service time of customer $i$

$t d_{i j}\left(t t_{i j}\right) \quad$ travel distance (time) from customer $i$ to customer $j$

$v c^{k} \quad$ variable travel cost associated with vehicle $k$

$f c^{k} \quad$ daily fixed cost for subcontracting vehicle $k$

$a_{i}^{v} \quad$ starting time of time window $v$ at customer $i$

$b_{i}^{v} \quad$ finishing time of time window $v$ at customer $i$

$d_{i} \quad$ demand of customer $i$

$d d \quad$ cost of having two drivers for the same vehicle $k$

ah allowed hours for each driver to work 


\section{Decision Variables}

$x_{i j}^{k} \quad$ takes on 1 , if arc $(i, j)$ is used by vehicle $k$ ( 0 otherwise)

$w_{i}^{k} \quad$ time at which the vehicle $k$ starts servicing vertex $i$

$u_{i}^{v} \quad$ takes on 1 , if customer $i$ is visited in time-window $v$ ( 0 otherwise)

$e^{k} \quad$ takes on 1 , if vehicle $k$ requires an extra driver ( 0 otherwise)

Let $\delta^{+}(i)=\{j:(i, j) \in A\}$ and $\delta^{-}(j)=\{i:(i, j) \in A\}$ denote the set of successors and predecessors of $i$ and $j$, respectively. The model $F_{d a y}$ for finding the optimal routes for customers serviced during the day is as follows:

$$
F_{d a y}=\min \sum_{(i, j) \in A} \sum_{k \in K_{i} \cap K_{j}} v c^{k} t d_{i j} x_{i j}^{k}+\sum_{k \in K} f c^{k}\left(1-x_{0, n+1}^{k}\right)+\sum_{k \in K} d d e^{k}
$$

subject to:

$$
\begin{gathered}
w_{n+1}^{k}-w_{0}^{k} \leq a h\left(e^{k}+1\right) \quad \forall k \in K \\
\sum_{k \in K_{i}} \sum_{j \in \delta^{+}(i)} x_{i j}^{k}=1 \quad \forall i \in N \\
\sum_{j \in \delta^{+}(0)} x_{0 j}^{k}=1 \quad \forall k \in K \\
\sum_{i \in \delta^{-}(j)} x_{i j}^{k}-\sum_{i \in \delta^{+}(j)} x_{j i}^{k}=0 \quad \forall j \in N, k \in K_{j} \\
\sum_{i \in \delta^{-}(n+1)} x_{i, n+1}^{k}=1 \quad \forall k \in K \\
w_{i}^{k}+s_{i}+t t_{i j}-w_{j}^{k} \leq b_{0}^{0}\left(1-x_{i j}^{k}\right) \quad \forall(i, j) \in A, k \in K_{i} \cap K_{j} \\
\sum_{v \in T W_{i}} u_{i}^{v} a_{i}^{v} \leq w_{i}^{k} \leq \sum_{v \in T W_{i}} u_{i}^{v} b_{i}^{v} \quad \forall i \in V, k \in K_{i} \\
\sum_{v \in T W_{i}} u_{i}^{v}=1 \quad \forall i \in V \\
\sum_{i \in N} \sum_{j \in \delta^{+}(i)} d_{i} x_{i j}^{k} \leq C^{k} \quad \forall k \in K \\
x_{i j}^{k}, u_{i}^{v}, e^{k} \in\{0,1\} ; \quad w_{i}^{k} \geq 0 .
\end{gathered}
$$

Objective function (1) minimizes the total cost involved in the daily routing. Since the fleet is completely outsourced it is possible to measure accurately the cost of the routing plan. Depending on the vehicle there is a variable cost related with the distance travelled, a fixed cost related with each vehicle used and, finally, a cost for having trips that are long enough to require two drivers.

In equation (2) the need for the extra driver is assessed through the allowed hours ah that each driver can work. Constraints (3) ensure that each customer is visited exactly once. Equations (4)-(6) establish the flow of each vehicle. Hence, every vehicle has to leave the depot and return to it by passing through the customers it is designed to serve. A vehicle can only 
start servicing a customer after having finished servicing the previous customer and after the time spent on traveling from the previous to the current customer (7). In equation (8) the start of the customer service is forced to be in between one of the multiple customer time windows. However, at each customer only one of the possible time windows may be used (9). Constraints (10) ensure that the different vehicle capacities are respected. To be more accurate both weight and volume constraints should exist in such model. However, due to the lack of reliable data, only weight requirements are considered (a practice also done by the company).

In order to model the problem of serving the night customers, a new parameter $n c$ to account for the extra-cost of having a driver at night has to be defined. In terms of the solution space, this problem is rather the same as the one for the day customers except for the fact that the night customers do not have multiple time windows and trips require no extra drivers. The night model $\left(F_{\text {night }}\right)$ is formulated as:

$$
F_{\text {night }}=\min \sum_{(i, j) \in A} \sum_{k \in K_{i} \cap K_{f}} v c^{k} t d_{i j} x_{i j}^{k}+\sum_{k \in K} n c\left(1-x_{0, n+1}^{k}\right)
$$

subject to:

$$
\begin{gathered}
(3)-(7),(10) \\
a_{i} \leq w_{i}^{k} \leq b_{i} \quad \forall i \in V, k \in K_{i} \\
x_{i j}^{k} \in\{0,1\} ; \quad w_{i}^{k} \geq 0 .
\end{gathered}
$$

The objective function of $F_{\text {night }}$ differs considerably from the one of $F_{\text {day }}$. In (12) we aim at minimizing the travel costs for the different vehicles, but the fixed costs are no longer dependent on the vehicle (since it was already paid for in the day shift) but just upon the night usage.

One could have opted for a single model to tackle the complete routing problem involving day and night customers. However, through the splitting of the models we achieve a considerable reduction in the problem size without jeopardizing optimality. Only in the case where $F_{d a y}$ does not contain the best trucks to be used at night, the decoupled models would not guarantee the same optimal solution as an integrated approach. However, due to the reasonably low heterogeneity of the fleet and the difference of magnitude between the number of day and night customers (day $>>$ night) this situation will never occur.

\section{Solution Method}

In order to solve various types of vehicle routing problems, a wide variety of solution methods have been developed. In our case, we want a flexible metaheuristic that is able to incorporate all the specificities of our problem and delivers reasonable results in short computation time. Gendreau and Potvin (2010) acknowledge that the adaptative large neighbourhood search (ALNS) framework introduced in Ropke and Pisinger (2006b) is able to obtain equal or better results for a wide variety of routing problems compared to existing algorithms. In Pisinger and Ropke (2007) very good results are reported for different classes of the VRP. Within the extensions tested some characteristics are coincident with features of our problem. The relevant problems are the VRP, the VRP-TW, and the SD-VRP.

Our algorithm is based on the ALNS developed by Kovacs et al (2012) for service technician routing and scheduling problems, incorporating site-dependent aspects due to skills and skill level requirements of the tasks. For specific details the readers are referred to this manuscript. 
For the sake of self-containedness we present here only the key blocks and ideas and the modifications we made with respect to the original algorithm.

Algorithm 1 outlines the ALNS framework. First, a feasible solution $s$ is generated. This solution may easily be generated because we allow some customers not to be serviced (unassigned customers included in set $N^{0}$ ) at a given penalty cost. In every iteration, a destroy-repair heuristic pair $(d, r)$ is chosen based on its respective score and weight obtained in previous iterations. Second, the destroy operator of the chosen pair is used to remove customers of the incumbent solution and places them into the set of unassigned customers. Third, the repair operator $r$ takes unassigned customers and inserts them into the routes. If the new solution $s^{\prime}$ meets the acceptance criteria, then it replaces $s$. If it is better than the best solution found so far, it replaces $s^{*}$. Finally, scores and weights $\left(\psi_{d r}\right.$ and $\left.\rho_{d r}\right)$ are updated and the algorithm proceeds to the next iteration. This is repeated until the stopping criterion is met.

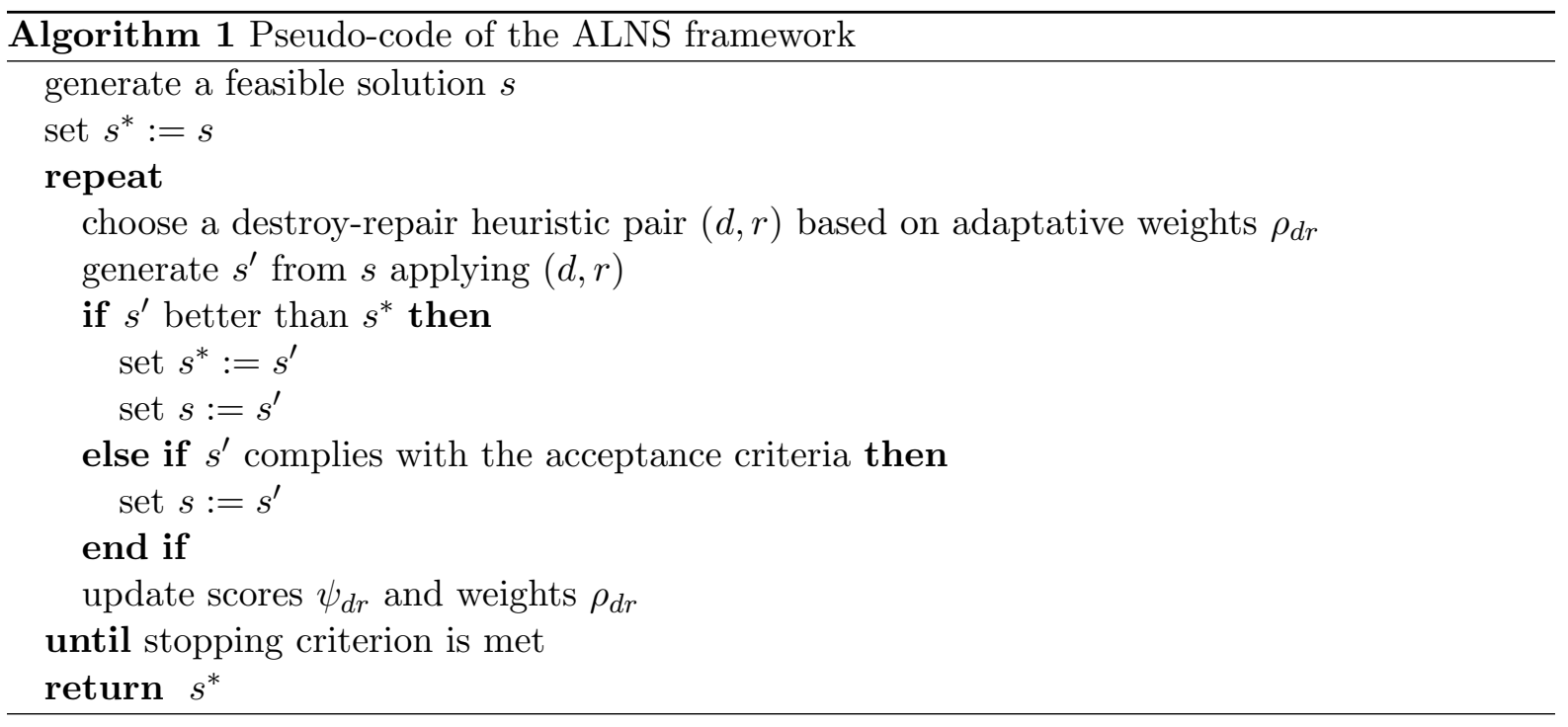

In the next subsections, the different destroy and repair operators, the used acceptance criteria, and how a destroy-repair heuristic pair is chosen are briefly presented.

\subsection{Destroy Operators}

Kovacs et al (2012) use four destroy operators, namely a random removal, a worst removal, a related removal, and a cluster removal operator. They are all based on operators introduced by Ropke and Pisinger (2006b) and Pisinger and Ropke (2007). In every iteration the number of customers $u$ to be removed from the different routes is chosen randomly from the interval $\left[0.1\left|N-N^{0}\right|, 0.4\left|N-N^{0}\right|\right]$.

The random removal operator removes $u$ customers randomly from their routes. The worst removal operator removes $u$ customers from the different routes biasing the selection towards customers that are not well inserted in terms of their distances from their current direct predecessor and successor customer locations. The related removal operator removes related requests. Since we consider vehicle capacities, we do not resort to the same relatedness measure as Kovacs et al (2012) but we use the relatedness measure of Pisinger and Ropke (2007), combining distance, time and load terms,

$$
R_{i j}=\alpha t d_{i j}+\beta\left|w_{i}-w_{j}\right|+\gamma\left|d_{i}-d_{j}\right| .
$$

Their respective weights are set to $\alpha=9, \beta=3, \gamma=4$ (Pisinger and Ropke, 2007). Finally, the cluster removal operator removes customers forming a cluster. Each selected route is split into two clusters through the computation of a minimum spanning tree where the longest arc is removed. Entire clusters are removed until the number of removed customers $\geq u$. 


\subsection{Repair Operators}

Following Kovacs et al (2012), we use six different insertion heuristics in terms of repair operators: a greedy insertion heuristic, four regret heuristics, and a sequential insertion heuristic. The greedy heuristic repeatedly inserts a customer from the set of unassigned customers at the cheapest feasible position. This is repeated either until all customers have been inserted or no more customers can be inserted maintaining feasibility. Regret heuristics improve the greedy heuristic described above, by integrating look ahead information when selecting customers to insert. Let $\Delta_{i}^{k}$ denote the change in the objective value for inserting customer $i$ at its best position in its $k$-cheapest route. In each iteration, the regret heuristic chooses the next customer $i$ to be insert as follows:

$$
i:=\arg \max _{i \in N^{0}}\left\{\sum_{k=2}^{\min (q, m)}\left(\Delta_{i}^{k}-\Delta_{i}^{1}\right)\right\}
$$

depending on the chosen value of $q$. We use $q \in\{2,3,4, m\}$. The parameter $m$ denotes the number of routes currently available for insertion. The sequential insertion heuristic corresponds to the I1 heuristic of Solomon (1987). It estimates the benefit coming from servicing a customer on the selected route rather than being serviced on a single customer tour.

Our objective function does not only incorporate distance based costs. The insertion of an additional customer into an existing route may also increase the number of necessary drivers. Therefore, we approximate the actual insertion costs by adding the costs for an additional driver in the case where the current duration of the route plus the additional time needed to service the respective customer exceeds the maximum duration of a single driver.

Furthermore, instead of a single time window at each customer location, we consider multiple time windows. Therefore, in order to check the feasibility of an insertion, we sequentially check time window feasibility at each customer that is serviced after the prospective insertion position of the new customer with respect to all available time windows.

\subsection{Acceptance Criteria}

As in Ropke and Pisinger (2006a), the destroy and repair operators are embedded into a simulated annealing framework. Hence, a solution $s^{\prime}$ is accepted if it is better than $s$. If $s^{\prime}$ is worse, $s^{\prime}$ replaces $s$ with a probability of $e^{\left(f\left(s^{\prime}\right)-f(s)\right) / \hat{t}}$. The parameter $\hat{t}$ denotes the current temperature.

\subsection{Choosing a destroy-repair heuristic pair $(d, r)$}

Instead of using separate scores and weights for each destroy and each repair operator, we use scores and weights for pairs of operators as in Kovacs et al (2012). Every combination out of the set of destroy and repair operators is used. The probability for choosing an operator pair is proportional to $\rho_{d r}$ for each destroy-repair pair $(d, r)$. To understand the computation of the weights, let us consider $n_{d}$ and $n_{r}$ as the respective number of destroy and repair heuristics implemented. First, the probability $\phi_{d r}$ of choosing a given pair is calculated as follows:

$$
\phi_{d r}=\frac{\rho_{d r}}{\sum_{d^{\prime}=1}^{n_{d}} \sum_{r^{\prime}=1}^{n_{r}} \rho_{d^{\prime} r^{\prime}}} .
$$

One pair is chosen in every iteration of the ALNS algorithm using roulette wheel selection. Based on the scores obtained, the weights are adjusted dynamically during the search. In the beginning, the weights $\rho_{d r}$ of all heuristic pairs are set to one and the scores $\psi_{d r}$ are set to zero. At the end of every iteration the scores $\psi_{d r}$ of the employed heuristic pair $(d, r)$ are updated as follows: $\psi_{d r}+\sigma_{1}$, if the destroy-repair heuristic pair gives a solution that improved the global best solution $s^{*} ; \psi_{d r}+\sigma_{2}$, if the destroy-repair heuristic pair gives a solution that was not visited before and improved the incumbent solution $s ; \psi_{d r}+\sigma_{3}$, if the destroy-repair heuristic pair gives a solution that was not visited before and was accepted as the new incumbent solution 
$s$, although it was worse; otherwise the value stays the same. Following Ropke and Pisinger (2006b) and Kovacs et al (2012), the parameters are set to $\sigma_{1}=33, \sigma_{2}=9$, and $\sigma_{3}=13$. Every 100 iterations, the weights are updated based on the current scores and the scores are reset to zero.

\section{Experimental Analysis and Comparison with Company's Prac- tice}

The company of our case study relies on a routing software to partially obtain their routes. This routing software is connected to the company's Enterprise Resource Planning (ERP) system and it receives all orders for the next day. It disposes of a database which contains the fixed customer data, such as geographical location, vehicle typologies and allowed time windows. To obtain a routing plan for the next day, the software is run for about 10 minutes and retrieves a solution that is then refined by the company's dispatching expert. The software uses as main optimization input a set of predefined routes defined by the planner based on his expertise.

Regarding our solution method, a main data problem regarding the travel times and distances was found. Since the routing software only had Cartesian coordinates for the customers and, unfortunately, we could not access its distance matrix, we recurred to Google Maps in order to calculate both travel times and distances. In that sense, a $\mathrm{C}++$ program that uses the Google Maps API was created in order to build the complete distance matrix. To feed the program the actual addresses of each client are used and we adopted as preference prioritizing motorways over small streets. The remaining data was simply compiled, because the company has the distribution operation completely outsourced and, therefore, vehicle costs, extra drivers, etc. were easily obtained and left no gap for subjective interpretations of the final results. This together with the use of real distances was very important in convincing the company of the validity of the generated plans.

Our decision support system is to replace the software used to determine the routing. The inputs are loosely the same as it is connected to the ERP to get all orders for each customer and it has an internal database, configurable through Excel, to feed static data, such as allowed customer time windows, customer addresses and vehicles availability. The planner has no options to configure and as the algorithm is run, he gets as output the generated routes with its operational and cost indicators. After a validation, this output data is fed back to the ERP so it can handle the next steps.

In order to compare the impact of our automatically generated solutions with the plans of the company we used two peak days from the high season. The first instance contains 350 customers to be serviced (see Figure 2) and the second one 366 (see Figure 3).

In Tables 1 and 2, the detailed results for the first instance according to the company's plans and to the plans generated by the ALNS (in about 10 minutes) are presented, respectively. Three key operational indicators are worth of notice. Firstly, the average capacity utilization rises in the generated plans from $74 \%$ to $86 \%$. Secondly, although each route takes more time to be completed in our plan, the average distance travelled per route decreases. Finally, a better utilization of the drivers' 8-hour working windows is notorious. In Figure 4 a graph plotting the different costs for both plans is given. The cost advantage that the automated routing generates is clear from the graph. From the three costs adding to the total cost: fixed vehicle costs, variable vehicle cost and extra driver cost, it is the variable vehicle cost that contributes the most to the obtained cost reduction. This fact relates very much to the gain of operational efficiency already mentioned regarding the decrease on the kilometres travelled by each truck.

In Figure 5 the total costs for both instances and both plans are plotted. Within these two instances the cost relation between the generated and the company's plans is stable. Overall, the ALNS plans were able to raise the vehicle utilization from $75 \%$ to $89 \%$ allowing for a decrease in the number of vehicles used. Since the company's plans were based on loosely fixed routes, 


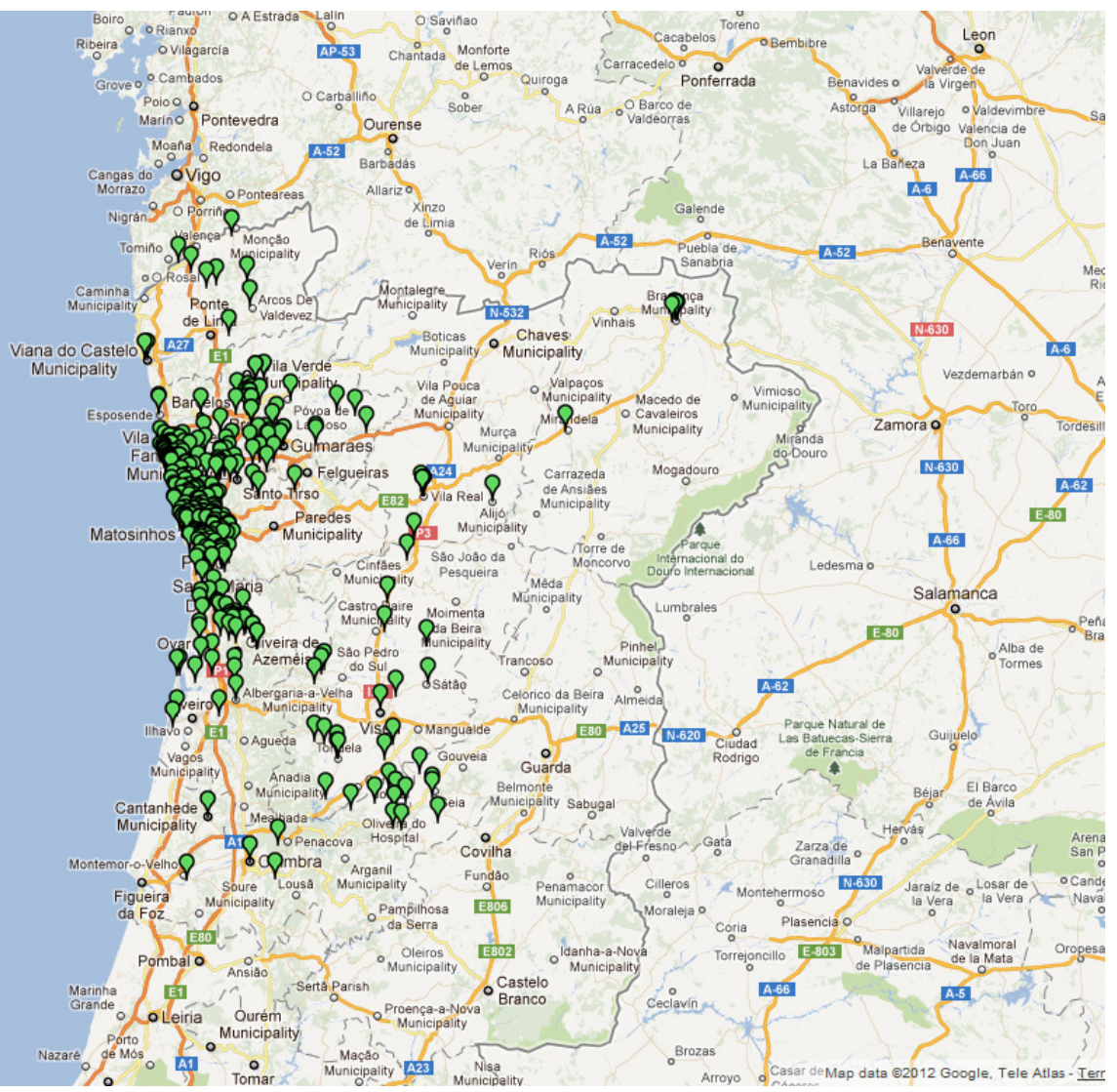

Figure 2: Geographical location of customers in the first instance

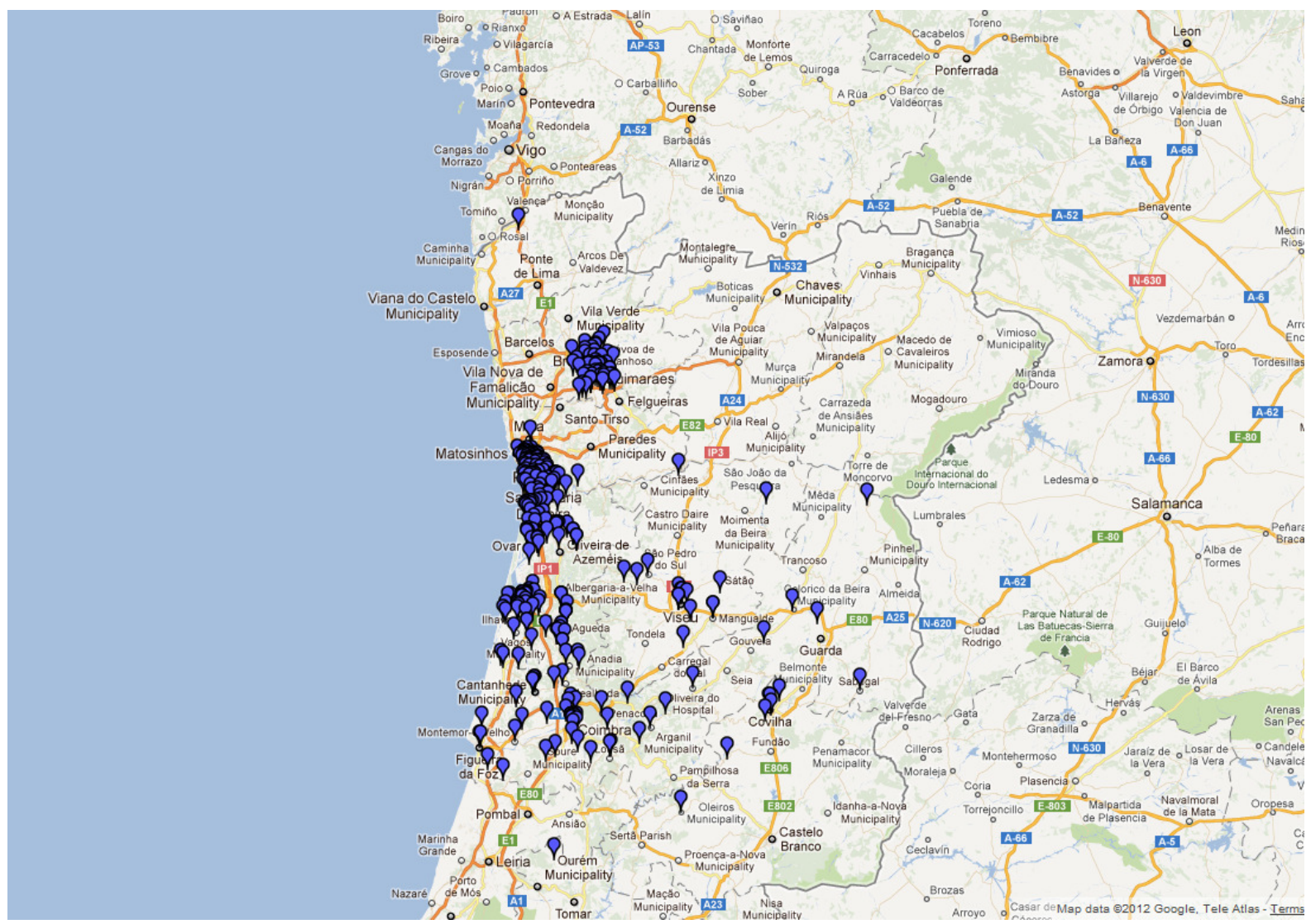

Figure 3: Geographical location of customers in the second instance 
Table 1: Detailed company's plan aggregated by route for the first instance.

\begin{tabular}{|c|c|c|c|c|c|c|c|c|c|}
\hline Route & 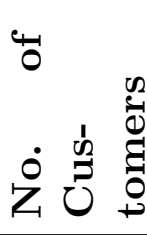 & 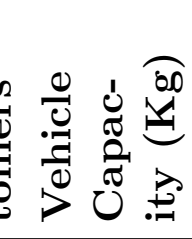 & 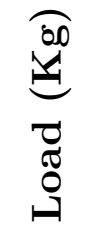 & 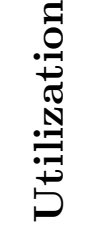 & 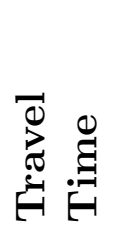 & 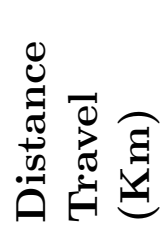 & 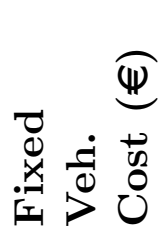 & 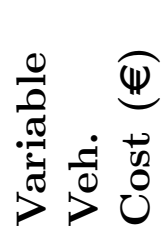 & 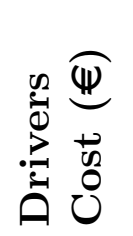 \\
\hline 1 & 22 & 8700 & 6821 & $78 \%$ & $6: 23$ & 183 & 138 & 93 & 0 \\
\hline 2 & 12 & 6500 & 5131 & $79 \%$ & $16: 05$ & 503 & 167 & 317 & 78 \\
\hline 3 & 14 & 6500 & 6051 & $93 \%$ & $12: 54$ & 403 & 167 & 254 & 78 \\
\hline 4 & 15 & 6500 & 5208 & $80 \%$ & $11: 21$ & 327 & 167 & 206 & 78 \\
\hline 5 & 9 & 6500 & 3721 & $57 \%$ & $7: 18$ & 286 & 178 & 109 & 0 \\
\hline 6 & 12 & 6500 & 5012 & $77 \%$ & $9: 28$ & 224 & 138 & 114 & 78 \\
\hline 7 & 12 & 6500 & 5241 & $81 \%$ & $8: 28$ & 204 & 167 & 129 & 78 \\
\hline 8 & 17 & 6500 & 5201 & $80 \%$ & 9:08 & 241 & 167 & 152 & 78 \\
\hline 9 & 18 & 6500 & 5464 & $84 \%$ & $9: 11$ & 222 & 167 & 140 & 78 \\
\hline 10 & 20 & 6500 & 4666 & $72 \%$ & $9: 14$ & 145 & 167 & 91 & 78 \\
\hline 11 & 15 & 6500 & 3129 & $48 \%$ & 9:02 & 122 & 178 & 47 & 78 \\
\hline 12 & 12 & 3950 & 3706 & $94 \%$ & $17: 11$ & 704 & 135 & 422 & 78 \\
\hline 13 & 13 & 3900 & 1587 & $41 \%$ & $10: 20$ & 442 & 135 & 265 & 78 \\
\hline 14 & 19 & 3900 & 2939 & $75 \%$ & $5: 37$ & 69 & 135 & 42 & 0 \\
\hline 15 & 23 & 3900 & 3560 & $91 \%$ & $6: 46$ & 61 & 135 & 37 & 0 \\
\hline 16 & 25 & 3950 & 3063 & $78 \%$ & $6: 11$ & 163 & 135 & 98 & 0 \\
\hline 17 & 23 & 4200 & 3200 & $76 \%$ & $7: 21$ & 151 & 165 & 83 & 0 \\
\hline 18 & 29 & 5450 & 3361 & $62 \%$ & $8: 22$ & 177 & 165 & 97 & 78 \\
\hline 19 & 26 & 3950 & 2472 & $63 \%$ & $6: 31$ & 107 & 135 & 64 & 0 \\
\hline 20 & 12 & 3950 & 3489 & $88 \%$ & $6: 43$ & 215 & 135 & 129 & 0 \\
\hline 21 & 2 & 2500 & 487 & $19 \%$ & $2: 14$ & 47 & 135 & 28 & 0 \\
\hline Average & 17 & (5398 & 3977 & $74 \%$ & $8: 50$ & $\begin{array}{c}238 \\
\text { TOTA }\end{array}$ & $\begin{array}{c}153 \\
\text { L COS }\end{array}$ & $\overline{139}$ & $\begin{array}{c}44 \\
€ 7050\end{array}$ \\
\hline
\end{tabular}


Table 2: Detailed plan generated by the metaheuristic aggregated by route for the first instance.

\begin{tabular}{|c|c|c|c|c|c|c|c|c|c|}
\hline Route & 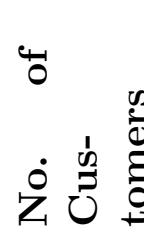 & 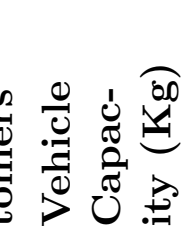 & 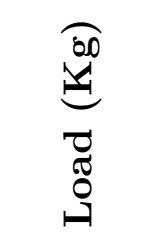 & 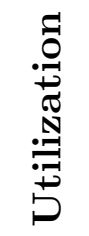 & 焉 & 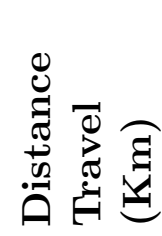 & 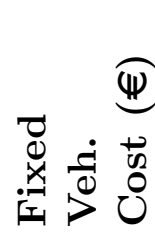 & 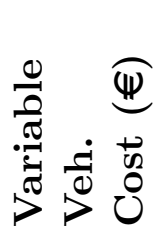 & 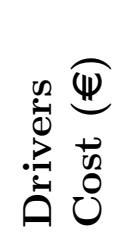 \\
\hline 1 & 8 & 6500 & 4996.88 & $77 \%$ & $7: 11$ & 58 & 167 & 37 & 0 \\
\hline 2 & 19 & 6500 & 6316.22 & $97 \%$ & 11:06 & 206 & 167 & 130 & 78 \\
\hline 3 & 19 & 6500 & 6298.05 & $97 \%$ & $12: 22$ & 349 & 167 & 220 & 78 \\
\hline 4 & 17 & 6500 & 5236.53 & $81 \%$ & 14:19 & 418 & 167 & 263 & 78 \\
\hline 5 & 7 & 6500 & 5629.71 & $87 \%$ & $7: 57$ & 191 & 167 & 120 & 0 \\
\hline 6 & 14 & 6500 & 6310 & $97 \%$ & $12: 09$ & 276 & 167 & 174 & 78 \\
\hline 7 & 24 & 3950 & 3478.43 & $88 \%$ & $7: 55$ & 113 & 135 & 68 & 0 \\
\hline 8 & 21 & 3950 & 3125.56 & $79 \%$ & $7: 57$ & 76 & 135 & 46 & 0 \\
\hline 9 & 19 & 3950 & 3436.37 & $87 \%$ & $7: 38$ & 143 & 135 & 86 & 0 \\
\hline 10 & 25 & 3950 & 3713 & $94 \%$ & $7: 40$ & 77 & 135 & 46 & 0 \\
\hline 11 & 23 & 3950 & 3836 & $97 \%$ & $7: 50$ & 258 & 135 & 155 & 0 \\
\hline 12 & 29 & 3950 & 2686 & $68 \%$ & $7: 54$ & 97 & 135 & 58 & 0 \\
\hline 13 & 18 & 3950 & 3857.22 & $98 \%$ & $7: 58$ & 127 & 135 & 76 & 0 \\
\hline 14 & 15 & 3950 & 3603.98 & $91 \%$ & $6: 43$ & 53 & 135 & 32 & 0 \\
\hline 15 & 20 & 3950 & 3389.69 & $86 \%$ & $7: 45$ & 100 & 135 & 60 & 0 \\
\hline 16 & 11 & 800 & 723.09 & $90 \%$ & $3: 57$ & 115 & 103 & 48 & 0 \\
\hline 17 & 15 & 5400 & 4576.97 & $85 \%$ & 10:17 & 217 & 165 & 119 & 78 \\
\hline 18 & 12 & 4200 & 3391.43 & $81 \%$ & $5: 44$ & 61 & 165 & 34 & 0 \\
\hline 19 & 17 & 6500 & 4382.13 & $67 \%$ & $19: 29$ & 430 & 178 & 163 & 78 \\
\hline 20 & 17 & 6500 & 5589.07 & $86 \%$ & $16: 19$ & 651 & 178 & 247 & 78 \\
\hline Average & 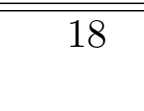 & 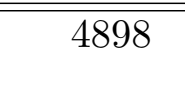 & $4 \quad 4229$ & $86 \%$ & "9:30 & $\begin{array}{c}201 \\
\text { TOT }\end{array}$ & $\begin{array}{c}150 \\
\text { L COS }\end{array}$ & 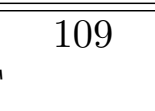 & $\begin{array}{c}27 \\
€ 5722\end{array}$ \\
\hline
\end{tabular}




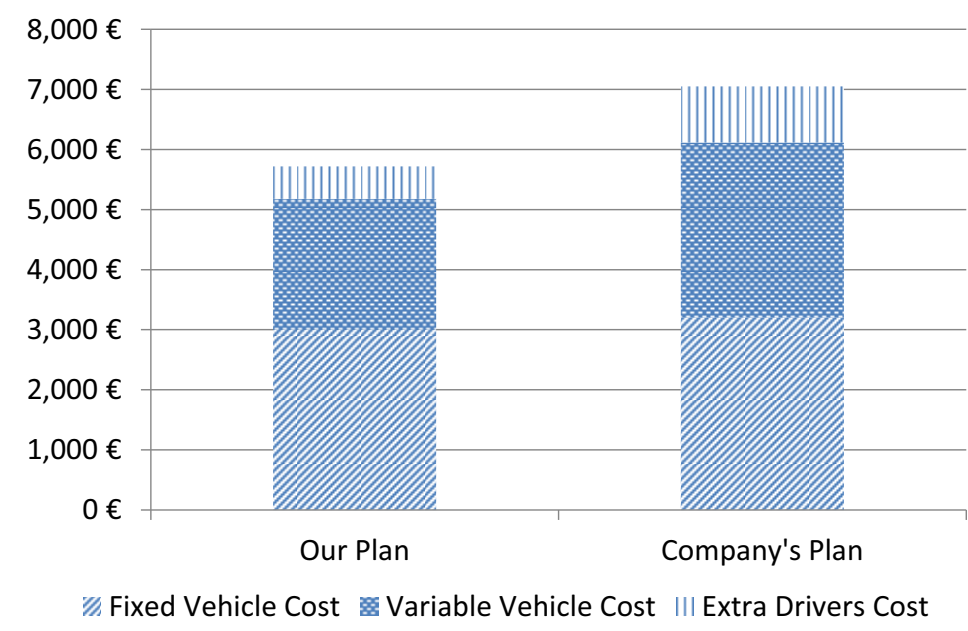

Figure 4: Comparison of both plans for all cost factors in the first instance.

demand consolidation in order to augment vehicle capacity utilization was harder to perform. The total distance travelled per day is reduced considerably in the order of 1200 kilometres. This is a very important achievement since variable vehicle costs account for most of the bill. Furthermore, with the increasing prices of oil derivatives the tendency is to see these costs rising in new contracts. Finally, we can expect that in the peak season the daily out-of-pocket savings can ascent to 1200 euros. By the end of the year these savings may have an interesting impact on the company's operating income, considering also the fact that the south filial of the company faces the same problem with a similar number of customers and a similar routing methodology.

Overall, the ALNS plans are able to reduce the consolidated costs per vehicle, which is a very important indicator for the company's top management, by almost $20 \%$ (from 350 to 286). Most of the cost reduction is achieved through a much better routing that consolidates more demand and delivers every product in a lower total distance. This kind of plan is hard to grasp and unveil by using common sense analysis.

The new plans have spillover effects that go beyond the cost reduction. Firstly, this methodology enforces every hard requirement to be met. From multiple time windows to site dependent vehicles, there are no operational constraints that are not respected. Previously, with the heavy manual planning some hard constraints were softened. This exigency and the extra flexibility achieved that allows an adaptation to increasingly changing market conditions, leverage customer satisfaction and, ultimately, customer loyalty. Secondly, the reduction on the amount of kilometres travelled per day may yield a better relationship with the third party logistics providers. The wear of the vehicles is to be reduced with this methodology and this may result in overall better results for these providers.

\section{Conclusions}

In this work we present a real world vehicle routing problem faced by a food distribution company in Portugal. The literature is rich in tackling sparse characteristics of these problems and, therefore, some of the extensions already addressed were gathered in order to formally 


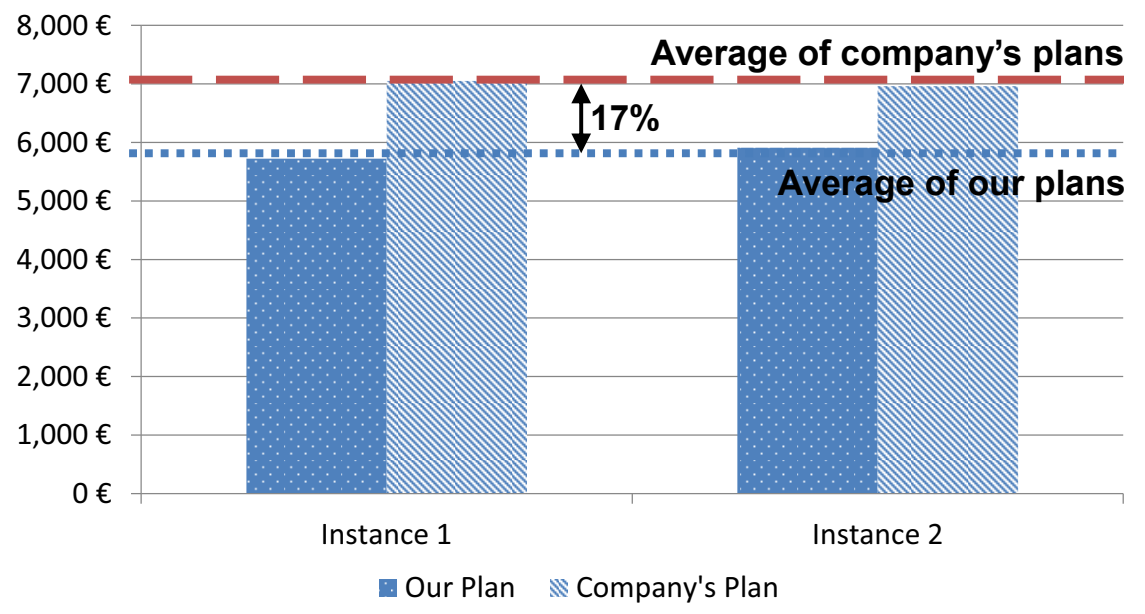

Figure 5: Comparison of both plans for the two instances.

identify the problem as a heterogeneous fleet site dependent vehicle routing problem with multiple time windows. Vehicle routing problems are known to be NP-hard and to solve real-world instances, metaheuristics are recommended. Since this specific problem has never been solved we chose to use a very general search procedure that has proven to deliver very good results for different vehicle routing related problems - the ALNS framework.

The direct impact of this tool is an average cost reduction for the company of about $17 \%$ in the distribution task at peak seasons. These savings are mainly achieved through a better capacity utilization of the vehicles and a reduction on the distance travelled to visit all customers. However, the advantages of the introduction of this decision support system go beyond the optimized routes. Hence, the related gains can be aligned in three axes: human resources optimization, improved reactivity of the company, stakeholders' satisfaction increase. The automatic decision support system provides a much better starting point for the planner's adjustments and reduces drastically the daily amount of time that this employee had allocated to such task. The tool is able to react to any new major development, such as the introduction of more customers. This ability is independent of the expertise of the user and may also allow the administration to simulate the operational impact of a major change, such as accepting to serve a client that is usually out of its acceptance range. Moreover, as every customer requirement is satisfied with certainty and the vehicles will be subject to less wear, an increase in stakeholder satisfaction is expected.

Since the tool is able to handle diverse routing extensions, it should be easy to roll-out to other companies facing similar real-world problems. Of course, the most straightforward step would be to go to other companies having a similar business model. Catering companies seem also a natural extension as they also handle different types of vehicles and exigent customers with several requirements. Nevertheless, the potential savings are of a lower magnitude as the amount and intensity of the deliveries is not the same. Other companies to which this approach could be rolled-out may be found in the waste collection business or big food producers that have their distribution process internalized.

Acknowledgments The first author appreciates the support of the Portuguese Science Foundation (FCT) Project PTDC/EGE-GES/104443/2008 and the FCT Grant SFRH/BD/ 68808/2010. 
The second author is supported by the Austrian Science Fund (FWF): T-514-N13. This support is gratefully acknowledged.

\section{References}

Ambrosino D, Sciomachen A (2006) A food distribution network problem: a case study. IMA Journal of Management Mathematics 18(1):33-53

Amorim P, Meyr H, Almeder C, Almada-Lobo B (2011) Managing perishability in productiondistribution planning: a discussion and review. Flexible Services and Manufacturing Journal pp 1-25, 10.1007/s10696-011-9122-3

Bitao P, Fei W (2010) Hybrid Intelligent Algorithm for Vehicle Routing Problem with Multiple Time Windows. In: 2010 International Forum on Information Technology and Applications, IEEE, pp 181-184

Chao IM, Golden B, Wasil E (1999) A computational study of a new heuristic for the sitedependent vehicle routing problem. INFOR 37(2):319-336

Chen HK, Hsueh CF, Chang MS (2009) Production scheduling and vehicle routing with time windows for perishable food products. Computers \& Operations Research 36(7):2311-2319

Cordeau J, Laporte G (2001) A tabu search algorithm for the site dependent vehicle routing problem with time windows. Infor-Information Systems and Operational Research 39(3):292298

Doerner K, Gronalt M, Hartl R, Kiechle G, Reimann M (2008) Exact and heuristic algorithms for the vehicle routing problem with multiple interdependent time windows. Computers \& Operations Research 35(9):3034-3048

Faulin J (2003) Applying MIXALG procedure in a routing problem to optimize food product delivery. Omega 31(5):387-395

Gendreau M, Potvin J (2010) Handbook of Metaheuristics, 2nd edn. Springer Science+Business Media

Hsu CI, Hung S, Li HC (2007) Vehicle routing problem with time-windows for perishable food delivery. Journal of Food Engineering 80:465-75

Instituto Nacional de Estatítica (2011) Estatísticas da produção industrial. http:// www . ine.pt/xportal/xmain?xpid\$=\$INE \\&xpgid\$=\$ine \textunderscorepublicacoes $\backslash$ \&PUBLICACOESpub $\backslash$ textunderscoreboui $\$=\$ 134140169 \backslash$ \&UBLICACOESmodo $\$=\$ 2$

Jansen D, Vorst G, Weert A (1998) Multi-compartment Distribution in the Catering Supply Chain. International Transactions in Operational Research 5(6):509-517

Kovacs A, N Parragh S, Doerner K, Hartl R (2012) Adaptive large neighborhood search for service technician routing and scheduling problems. Journal of Scheduling 15(5):579-600

Mullaseril P, Dror M, Leung J (1997) Split-Delivery Routeing Heuristics in Livestock Feed Distribution. The Journal of the Operational Research Society 48(2):107-116

Nag B, Golden B, Assad A (1998) Vehicle routing with site dependencies. In: Golden B, Assad A (eds) Vehicle routing: methods and studies, Elsevier, Amsterdam, pp 149 - 159

Osvald A, Stirn L (2008) A vehicle routing algorithm for the distribution of fresh vegetables and similar perishable food. Journal of Food Engineering 85(2):285-295 
Pisinger D, Ropke S (2007) A general heuristic for vehicle routing problems. Computers \& Operations Research 34(8):2403-2435

Ropke S, Pisinger D (2006a) A unified heuristic for a large class of Vehicle Routing Problems with Backhauls. European Journal of Operational Research 171(3):750-775

Ropke S, Pisinger D (2006b) An Adaptive Large Neighborhood Search Heuristic for the Pickup and Delivery Problem with Time Windows. Transportation Science 40(4):455-472

Savelsbergh M (1985) Local search in routing problems with time windows. Annals of Operations Research 4(1):285-305

Solomon M (1987) Algorithms for the vehicle routing and scheduling problems with time window constraints. Operations research 35(2):254-265

Tarantilis C, Kiranoudis C (2001) A meta-heuristic algorithm for the efficient distribution of perishable foods. Journal of Food Engineering 50(1):1-9

Tarantilis C, Kiranoudis C (2002) Distribution of fresh meat. Journal of Food Engineering 51(1):85-91 